\title{
Muerte encefálica: repercusión sobre órganos y tejidos
}

\author{
J.M. Domínguez-Roldán*, C. García-Alfaro, P.I. Jimenéz-González, \\ F. Hernández-Hazañas, \\ M.L. Gascón Castillo y J.J. Egea Guerrero
}

Unidad de Gestión Clínica de Cuidados Críticos y Urgencias, Hospital Universitario Virgen del Rocío, Sevilla, España

Recibido el 17 de febrero de 2009; aceptado el 5 de marzo de 2009

Disponible en Internet el 24 de septiembre de 2009

\section{PALABRAS CLAVE \\ Muerte encefálica; \\ Citoquinas; \\ Inflamación; \\ Trasplante; \\ Donación de órganos; \\ Disfunción del injerto; \\ Rechazo.}

\section{KEYWORDS}

Brain death;

Cytokines;

Inflammation;

Transplantation;

\begin{abstract}
Resumen
La muerte encefálica se acompaña de una serie de efectos sistémicos, hemodinámicos, hormonales e inflamatorios que tienen una repercusión relevante en los órganos y los tejidos de la economía. Cada vez hay más evidencias de que los órganos provenientes de donantes fallecidos en muerte encefálica presentan un grado de respuesta inflamatoria secundaria al daño encefálico y, en ocasiones, proporcional a la intensidad y a la velocidad de progresión de éste. Tanto estudios clínicos como estudios experimentales han mostrado que el resultado de los órganos de donantes fallecidos en parada cardíaca o donantes vivos tienen iguales o mejores resultados clínicos que los obtenidos en donantes en muerte encefálica que han presentado el proceso inflamatorio secundario a ésta. Hay pruebas de que esta respuesta inflamatoria acontece en el pulmón, el corazón, los riñones, el hígado y el intestino, e igualmente se incrementan también las pruebas de que el grado de respuesta inflamatoria observada en los órganos tiene una influencia importante en el resultado final del trasplante. En consecuencia, el desarrollo del conocimiento de las vías que interrelacionan el daño encefálico con la respuesta orgánica inflamatoria abre una importante área de conocimiento y posibilita que futuras estrategias terapéuticas encaminadas a modular la respuesta sistémica al daño encefálico permitan mejorar la calidad de los órganos obtenidos para trasplante, así como incrementar la supervivencia del injerto y de los receptores de trasplantes de órganos sólidos.
\end{abstract}

(c) 2009 Elsevier España, S.L. y SEMICYUC. Todos los derechos reservados.

Brain death: Repercussion on the organs and tissues

\section{Abstract}

Brain death is accompanied by a series of hemodynamic, hormonal and inflammatory systemic effects that have an important repercussion on the economy of the organs and

\footnotetext{
*Autor para correspondencia.

Correo electrónico: jmdominguez@telefonica.net (J.M. Domínguez-Roldán).
} 
Organ donation; Organ dysfunction; Organ rejection. tissues. There is increasing evidence that the organs from brain death donors have an inflammatory response grade secondary to brain death and sometimes proportional to the intensity and rate of its progression. Both clinical and experimental studies have shown that the result of organs from heart arrest deceased donors or live donors have the same or better clinical results than those obtained in brain death donors and who have suffered the inflammatory process secondary to it. There is proof that this inflammatory response occurs in the lung, heart, kidneys, liver, intestine. Furthermore, the evidence also shows that the grade of inflammatory response observed in the organs has an important influence on the final outcome of the transplant. Consequently, the development of the knowledge regarding the pathways that interrelate brain death with the inflammatory organ response provides us with an important area of knowledge, which allow for future therapeutic strategies aimed at modulating the systemic response to brain death to improve the quality of the organs obtained for transplant and also to increase graft survival of the solid organ transplant recipients.

(c) 2009 Elsevier España, S.L. and SEMICYUC. All rights reserved.

\section{Introducción}

Actualmente, la mayor parte de los programas de donación y trasplante de órganos se sustentan en los órganos provenientes de los donantes en muerte encefálica. Se han estudiado diversos aspectos médicos durante los últimos años a fin de establecer cuáles son los factores más relevantes que influyen en el éxito de un trasplante. Durante años, se prestó una gran atención a la compatibilidad histológica donante/receptor e igualmente a la importancia que tenían los tiempos de isquemia en la posterior funcionalidad del órgano tras el trasplante. Igualmente, durante los primeros tiempos de los programas de trasplante se otorgó gran relevancia a un adecuado mantenimiento hemodinámico del donante en muerte encefálica, dada la reconocida influencia positiva que un buen mantenimiento del donante tenía sobre los resultados finales del trasplante. En los últimos tiempos un nuevo campo de mejora ha aparecido en el mundo de la trasplantología: el conocimiento de la importancia que la muerte encefálica tiene como factor generador de daño del órgano donado. En la última década ha comenzado a ponderarse la influencia que el daño encefálico tiene sobre los diversos órganos de la economía, y por esto también se ha empezado a reconocer la influencia que esos cambios en los órganos donados (consecuencia de la muerte encefálica) pueden tener en el resultado del injerto y la supervivencia del receptor.

Algunos estudios a mediados de los años 70 probaron que la tasa de supervivencia de los riñones provenientes de donantes vivos no emparentados era comparable a la supervivencia de los riñones provenientes de donantes vivos que compartían un haplotipo, y era superior a aquellos injertos provenientes de cadáveres en muerte encefálica matcheados ${ }^{1}$. Esa diferencia podía explicarse, en parte, por el aumento de la función retrasada del injerto renal proveniente de donantes en muerte encefálica y que aumentan el tiempo y la intensidad de la respuesta alogénica del huésped ${ }^{2}$, pero por otra, por los cambios ocurridos en el órgano antes del injerto.

Por otra parte, existen evidencias de que los riñones de donantes a corazón parado pueden tener superiores resultados en el trasplante cuando se comparan con los riñones de donantes en muerte encefálica. Hace más de 15 años, Orloff et $\mathrm{al}^{3}$ pusieron en evidencia, tras el seguimiento durante años de pacientes que habían recibido órganos de donantes en muerte encefálica y de donantes a corazón parado, que estos últimos presentaban un significativo menor nivel de creatinina plasmática, unos requerimientos de diálisis postoperatoria menor y una supervivencia del injerto significativamente mayor. Hace más de una década comenzaron a presentarse pruebas de que la muerte encefálica se acompaña de una serie de cambios funcionales en los riñones del donante, que pueden influir en la viabilidad del órgano una vez trasplantado.

Una interesante y reciente publicación ${ }^{4}$ analizó la supervivencia de receptores de órganos provenientes de un grupo controlado de donantes en los que se había realizado una evaluación de la respuesta inflamatoria (TNF, interleuquina- 6 e interleuquina-10 en el momento del desarrollo de la muerte encefálica y posteriormente cada $4 \mathrm{~h}$ hasta la extracción de órganos) a fin de conocer su posible influencia en el resultado final del trasplante. Este estudio pudo confirmar, en todos los donantes, que los niveles de citoquinas estaban significativamente elevados, además de ello, las altas concentraciones plasmáticas de interleuquina6 en los donantes se asociaron de modo significativo a una disminución de la supervivencia del receptor a los 6 meses de su alta hospitalaria. Estos hallazgos clínicos indican que existen diversos factores inmunológicos y no inmunológicos que pueden influir en el resultado final del trasplante ${ }^{5,6}$.

La muerte encefálica no debe considerarse como una situación estática, sino como un proceso dinámico que de un modo directo y a través de múltiples mecanismos puede tener una influencia significativa en la calidad de los órganos donados. Parece, por tanto, evidente que la muerte encefálica puede considerarse como el mayor factor de estrés al que se someten los órganos antes del trasplante y que es inductora de una importante respuesta inflamatoria (tabla 1); actualmente es campo de debate cuál es la relevancia que la respuesta inflamatoria puede tener sobre la viabilidad de órganos para trasplante y la supervivencia del injerto. Por otra parte, y de acuerdo con Kusaka et $\mathrm{al}^{7}$, los órganos alogénicos trasplantados, especialmente los provenientes de cadáveres subóptimos en muerte encefálica, pueden no ser biológicamente inertes en el 
Tabla 1 Algunos de los fenómenos inflamatorios orgánicos que se han descrito tras la muerte encefálica

Expresión de ICAM-1 en el riñón y el hígado

Infiltrado leucocitario en el riñón

Expresión de interleuquina-1- $\beta$ e interleuquina- 6 en el riñón

Incremento del coeficiente de ultrafiltración en el pulmón

Elevación de los niveles plasmáticos de interleuquina-1- $\beta$, TNF- $\alpha$, IFN- $\gamma$ y CINC-3

Expresión del ARNm de la interleuquina-8 en el pulmón

Infiltración leucocitaria en el hígado

Sobrerregulación de ICAM-1, E-selectinas y VCAM-1 en el intestino

CINC: cytokine-induced neutrophil chemoattractant; ICAM-1: moléculas de adhesión intercelular; IFN- $\gamma$ : interferón gamma; mARN:

ARN mensajero; TNF- $\alpha$ : factor de necrosis tumoral alfa; VCAM-1: moléculas de adhesión vascular.

momento de su implantación y podrían estar programados para iniciar o amplificar una respuesta inflamatoria por parte del huésped. Esta potencial activación de los órganos puede representar un contínuum entre los cambios inflamatorios secundarios a una agresión general inespecífica y el posterior desarrollo de una puesta inmunológica ${ }^{8}$.

Existe actualmente una renovada opinión de que desde el momento en que se declara la muerte encefálica y se produce la extracción de órganos existe un período en que pueden producirse lesiones progresivas de los órganos debidas a la muerte encefálica. Por esto, podría establecerse una estrategia de citoprotección e histoprotección para disminuir los efectos negativos de la muerte encefálica durante este período. Las propuestas terapéuticas en el donante de órganos se han ido ampliando en los últimos años. Los protocolos iniciales se dirigían especialmente al control de la hipotensión generada tras la muerte encefálica y al mantenimiento de los electrólitos y el agua intravascular en rangos normales, posteriormente se incorporó el tratamiento hormonal sustitutivo, fundamentalmente con hormonas tiroideas ${ }^{9}$. Actualmente, se han puesto no sólo tratamientos genéricos, sino también tratamientos organoespecíficos para mejorar la viabilidad de los órganos que van a trasplantarse. Así, por ejemplo, se ha propuesto la cardioprotección mediante el empleo de betabloqueantes para disminuir la miocitolisis que se presenta en el corazón de los donantes ${ }^{10}$. En los últimos años, el incremento del conocimiento de la fisiopatología de los episodios que ocurren tras la muerte encefálica ha posicionado nuevas opciones terapéuticas como altamente recomendadas para el tratamiento del donante de órganos, entre las que se incluyen tratamientos con esteroides o estrategias inmunomoduladoras. En cualquier caso, sí parece evidente que los iniciales conceptos de "mantenimiento del donante", que posteriormente dieron paso al concepto de "manejo del donante", han cambiado y actualmente debemos hablar de "tratamiento del donante de órganos", que debe ir dirigido al incremento de la viabilidad de los órganos trasplantados y al incremento de la supervivencia de injertos y receptores.

\section{Influencia de la muerte encefálica sobre el corazón}

El incremento de la presión intracraneal es el mecanismo más frecuentemente vinculado al desarrollo de muerte encefálica. Las relaciones entre este proceso y el daño de los órganos intratorácicos se han estudiado tanto a nivel clínico como a nivel experimental ${ }^{11}$, y puede actualmente establecerse una directa relación entre este fenómeno y el daño cardíaco que se presenta en los donantes.

Existen diversas evidencias que prueban que la elevación de la presión intracraneal desencadenante de la muerte encefálica se asocia, de modo evidente, tanto a cambios histológicos en el miocardio del donante como a trastornos de la funcionalidad cardíaca. Estudios experimentales han probado que el incremento súbito de la presión intracraneal se asocia al desarrollo de una relevante respuesta hiperdinámica cardiocirculatoria y a un incremento de los niveles de epinefrina. Shivalkar et $\mathrm{al}^{12}$ pudieron observar que los niveles de aminas se incrementaban de modo mucho más significativo cuando la elevación de la presión intracraneal era súbita que cuando ese incremento era progresivo. Igualmente observaron que cuando se trasplantaron corazones de animales que habían fallecido por un incremento súbito de la presión intracraneal, la funcionalidad miocárdica era inferior a la detectada en corazones de animales en los que la muerte encefálica se generó mediante un incremento progresivo de la presión intracraneal. También pudieron establecer que la función miocárdica de este último grupo era inferior a la de aquellos corazones trasplantados de animales donantes que no habían tenido el proceso de muerte encefálica.

La influencia que la actividad simpática, generada durante el proceso de la muerte encefálica, tiene sobre la función miocárdica pudo confirmarse mediante la cuantificación de los niveles de norepinefrina (a través de un sistema de microdiálisis) simultáneamente a la monitorización de la presión intracraneal en un modelo de hipertensión intracraneal y muerte encefálica. La aparición progresiva de daño encefálico (elevación experimental de la presión intracraneal) hasta la muerte encefálica se asoció a un muy significativo incremento de la concentración de norepinefrina en un fenómeno de liberación bifásico, con un pico inmediatamente después de la hipertensión intracraneal y la muerte encefálica y un segundo pico de concentración 40 min después de ésta, lo que confirma el incremento masivo de liberación de norepinefrina en las terminaciones nerviosas miocárdicas tras la muerte cerebral. Mertes et $\mathrm{al}^{13}$ y Ferrera et $\mathrm{al}^{14}$ encontraron hallazgos similares sobre una respuesta bifásica en estudios experimentales en muerte encefálica (el segundo pico se situó 60 min después de la muerte encefálica), también 
pudieron constatar incrementos proporcionales de la presión arterial que correspondían en paralelo a la evolución de los niveles de catecolaminas. Estos autores también estudiaron la influencia que otras alteraciones hormonales podían tener sobre la funcionalidad miocárdica durante el proceso de la muerte encefálica y confirmaron que durante el período inicial de inducción de la muerte encefálica, los niveles de triiodotironina y levotiroxina, prolactina y melatonina no se alteraban de modo significativo, lo que indica una estabilidad de las hormonas tiroideas en contraste con las alteraciones de la corteza y la médula suprarrenal, que sí presentaban significativas alteraciones durante este período.

En un modelo experimental que analizó las sobreexpresión del mARN de moléculas inflamatorias en el corazón de ratas en muerte encefálica ${ }^{15}$, pudo evidenciarse que, en comparación con el grupo control (no en muerte cerebral), existía una expresión significativamente más evidente del mARN de moléculas de adhesión intercelular (ICAM-1) y de moléculas de adhesión vascular (VCAM-1), de interleuquina-1- $\beta$ y de interleuquina-6. Las diferencias encontradas entre ambos grupos no pudieron atribuirse a los fenómenos hemodinámicos (hipertensión e hipotensión), frecuentemente observados en muerte encefálica, ya que en este estudio se mantuvo una completa estabilidad hemodinámica durante todo el experimento. En este modelo experimental no se observaron diferencias en la expresión de mARN, TNF- $\alpha$, IFN- $\gamma$, interleuquina-1- $\alpha$, interleuquina- 2 , interleuquina-2R- $\beta$ e interstitial monocyte chemoattractant 1 (IMCP-1).

\section{Influencia de la muerte encefálica sobre el pulmón}

El daño pulmonar que frecuentemente se observa en pacientes neurológicos críticos con hipertensión intracraneal y daño encefálico tiene, probablemente, aspectos comunes con la lesión pulmonar observada en donantes de órganos en muerte encefálica. Los mecanismos generadores de la disfunción temprana del injerto pulmonar se han estudiado ampliamente y se les ha atribuido un origen multifactorial. Diversos modelos experimentales y clínicos han demostrado la influencia que en la disfunción pulmonar postrasplante tiene la lesión por isquemia/reperfusión y la preservación pulmonar. Además de ello, diversas observaciones clínicas han venido a indicar cómo otros factores, como por ejemplo la influencia de la muerte encefálica sobre el pulmón, podían desempeñar también un papel en la disfunción primaria del injerto pulmonar. Es bien conocido que el daño neurológico grave y la muerte encefálica se asocian frecuentemente a alteraciones en el intercambio gaseoso y a lesión pulmonar aguda. También se ha demostrado que la lesión cerebral grave se asocia a un incremento en los niveles de interleuquina-8 en el líquido de lavado broncoalveolar.

El desarrollo de procesos inflamatorios tiene una gran importancia en los resultados clínicos del trasplante pulmonar, de hecho, no es infrecuente que los receptores de trasplantes pulmonares provenientes de donantes en muerte encefálica desarrollen bronquiolitis obliterante, una manifestación de rechazo crónico. Zweers et $\mathrm{al}^{16}$ realizaron investigaciones experimentales en animales a los que se les generaba un incremento de la presión intracraneal hasta desarrollar muerte encefálica, y pusieron en evidencia que en los pulmones de estos animales se generaba un significativo flujo de leucocitos, una expresión de moléculas de adhesión celular y una expresión del mARN de citoquinas. Cuando estos pulmones se trasplantaron y se examinaron después del trasplante (se compararon con un grupo control en el que se trasplantaron pulmones donados por animales que no se encontraban en muerte encefálica), se comprobó que la función pulmonar era significativamente peor en el grupo de pulmones provenientes de animales en muerte encefálica que en el grupo control. Otro hallazgo interesante fue que los estudios histológicos de los órganos mostraron signos más obvios de rechazo, incluyendo grave hiperplasia intimal. El mARN de la interleuquina-2 estuvo también significativamente elevado en los injertos pulmonares provenientes de donantes en muerte encefálica en comparación con el grupo control.

López-Aguilar et $\mathrm{al}^{17}$ desarrollaron un interesante estudio para analizar la influencia del daño cerebral masivo en la génesis de la lesión pulmonar secundaria a ésta. Para esto, indujeron la muerte encefálica a un grupo de animales mediante la inserción de un catéter intracraneal que generaba un importante incremento de la presión intracraneal. En la fase in vivo no se detectaron cambios relevantes en el intercambio gaseoso ni en la mecánica pulmonar cuando se compararon ambos grupos. En la fase ex vivo, los pulmones de animales con daño cerebral masivo desarrollaron mayor grado de edema (evaluado mediante ganancia de peso) que el grupo control; el coeficiente de ultrafiltración pulmonar se incrementó en ambos grupos, aunque en modo significativamente mayor en el grupo de lesión cerebral masiva. En este grupo, la puntuación de daño histológico fue superior que en el grupo control. Así, la hemorragia alveolar (definida como el número de alvéolos que contienen eritrocitos) y la hemorragia perivascular (definida como el número de vasos extraalveolares que estaban rodeados por hemorragia perivascular prominente) fueron también mayores en el grupo con lesión cerebral masiva que en el grupo control.

Bajo la hipótesis de que los cambios hemodinámicos que se producen durante la muerte encefálica son causantes, en gran parte, de la respuesta inflamatoria de los pacientes en muerte encefálica, Avlonitis et $\mathrm{al}^{18}$ desarrollaron estudios experimentales de muerte encefálica en que la respuesta hemodinámica se moduló a fin de controlar la hipertensión y la hipotensión arterial que frecuentemente se presenta tras el desarrollo de ésta. La inducción de la muerte encefálica sin ningún tipo de control hemodinámico condicionó una significativa elevación de los niveles plasmáticos de citoquinas proinflamatorias y de quimioquinas (interleuquina-1$\beta$, TNF- $\alpha$ y CINC [cytokine-induced neutrophil chemoattractant] 3) y un incremento de la expresión de las integrinas CD11b y CD18 de los neutrófilos. También se observó un deterioro de la oxigenación y un significativo incremento, comparado con el grupo control, de interleuquina-1- $\beta$, TNF$\alpha$, CINC-1 y CINC-3, en el líquido de lavado broncoalveolar. Llamativamente, estos últimos hallazgos (alteraciones de la oxigenación e incremento de citoquinas) pudieron prevenirse cuando a los animales de experimentación se les administró tratamiento con fentolamina. Estos últimos datos 
pueden llevar a inferir que la lesión inflamatoria pulmonar observada tras la muerte encefálica está causada, al menos en parte, por un estrés hemodinámico producido durante la tormenta catecolaminérgica, lo que podría también explicar en cierto grado la propuesta de tratamiento del edema pulmonar neurogénico mediante esteroides que algunos autores han realizado ${ }^{19,20}$. El estudio mediante microscopia electrónica del tejido pulmonar en el grupo de muerte encefálica sin tratamiento y en el grupo tratado con noradrenalina reveló áreas de rotura de la membrana alveolocapilar, hallazgos que no aparecieron en el grupo protegido farmacológicamente con fentolamina. El tratamiento con noradrenalina (para evitar el episodio de hipotensión arterial asociado a la muerte encefálica) se asoció a unos niveles séricos más bajos de citoquinas/ quimioquinas y a una expresión de CD11b/CD18B más baja en comparación con el grupo no tratado.

También a nivel clínico se ha podido evidenciar la relación daño encefálico grave/lesión pulmonar. En un estudio clínico desarrollado sobre donantes pulmonares y sus correspondientes trasplantes, se estudiaron los niveles de interleuquina-8 en el líquido de lavado broncoalveolar de los pulmones donados y su potencial influencia en la posterior evolución del injerto ${ }^{21}$, además de los niveles de growthregulated oncogene-alfa $(G R O-\alpha)$, epithelial neutrophilactivating peptide 78 (ENA-78) y la expresión del mARN de la interleuquina-8 (en el tejido pulmonar del donante). En el líquido de lavado broncoalveolar del donante, se observó una elevación del recuento de neutrófilos con respecto a los valores considerados normales en individuos sanos. Existió también una relación directa entre el porcentaje de neutrófilos y la concentración de interleuquina-8 en este líquido. No existió relación entre el mecanismo de daño encefálico y la concentración de interleuquina-8 (daño traumático versus enfermedad cerebrovascular), ni tampoco con la duración de la ventilación mecánica que recibió el donante. La evolución clínica del injerto pulmonar presentó una relación con la concentración de interleuquina-8 en el lavado broncoalveolar de los donantes, y estuvo significativamente más elevada en aquellos pulmones que una vez trasplantados tenían peores índices de oxigenación. Los receptores que presentaron disfunción grave del injerto coincidían con aquellos que recibieron pulmones en los que existían altas concentraciones de interleuquina-8. Aquellos receptores que fallecieron tempranamente recibieron pulmones con una concentración de interleuquina- 8 significativamente más elevada que aquellos receptores que sobrevivieron más allá de 6 meses. La expresión del mARN de la interleuquina- 8 en el pulmón donante fue sustancialmente más elevada en los pulmones que desarrollaron disfunción grave del injerto que en aquellos que funcionaron bien después del tratamiento. En este mismo estudio pudo observarse cómo las concentraciones medias de ENA-78 y GRO- $\alpha$ en el líquido de lavado broncoalveolar eran superiores, aunque de modo no estadísticamente significativo, en receptores con disfunción temprana del injerto en comparación con los que presentaron una buena evolución en la fase inmediata postrasplante. En preparaciones de lavado broncoalveolar de donantes pulmonares que evolucionaron con una pobre función pulmonar postrasplante, cuyos receptores murieron en la fase temprana, la tinción específica para interleuquina- 8 fue intensa y distribuida por todo el citoplasma de los macrófagos alveolares, mientras que en receptores con buena función pulmonar, la tinción fue significativamente más débil y limitada a la región perinuclear. Ello indica que los macrófagos alveolares y las células epiteliales fueron las fuentes principales de aumento de interleuquina- 8 en los donantes pulmonares que presentaron una peor función pulmonar.

\section{Influencia de la muerte encefálica sobre el riñón}

El efecto deletéreo que la muerte encefálica tiene sobre los órganos donados tiene bases no solamente fisiopatológícas, sino también clínicas y epidemiológicas. Cuando se compararon los resultados del trasplante de riñones provenientes de pacientes fallecidos por parada cardíaca con aquellos que provenían de donantes en muerte encefálica, se pudo observar cómo no solamente tenían una similar supervivencia del injerto, sino que también se observó que en el grupo de riñones provenientes de donantes en muerte encefálica la función retrasada del injerto presentaba una menor incidencia 22,23 . Por otra parte, diversas investigaciones clínicas han mostrado que los injertos renales provenientes de donantes renales en muerte encefálica presentan episodios de rechazo agudo más frecuentes que aquéllos provenientes de donantes vivos ${ }^{24}$.

Relevantes estudios, como los de Kusaka et $\mathrm{al}^{7}$, han puesto en evidencia la significativa activación de mediadores inflamatorios en el injerto renal de riñones procedentes de animales en muerte encefálica. En los riñones injertados procedentes de animales que habían tenido una muerte encefálica experimental, se pudieron detectar P-selectinas, E-selectinas y componente C3 del complemento en el endotelio vascular y los glomérulos renales. La expresión de estas moléculas estuvo asociada a la presencia de leucocitos polimorfonucleares que infiltraban progresivamente el tejido renal, con un incremento progresivo durante las primeras $24 \mathrm{~h}$ después del trasplante. A los 5 días tras el injerto, se produjo una intensa expresión de citoquinas y moléculas inflamatorias en los túbulos y una infiltración de poblaciones leucocitarias en los túbulos y los glomérulos renales de los riñones que se habían expuesto a la muerte encefálica. En contraste, los órganos injertados de animales donantes vivos permanecían virtualmente con una expresión normal de citoquinas e infiltrados celulares. La citoquina proinflamatoria interleuquina- $\beta$ estaba significativamente elevada en el grupo de receptores que recibieron un riñón de donantes en muerte encefálica en comparación con los que recibieron un riñón proveniente de donante vivo.

Estudios clínicos en humanos sobre riñones trasplantados provenientes de donantes en muerte encefálica y donantes vivos $^{25}$ también evidenciaron resultados similares a los anteriormente comentados. Cuando se compararon donantes de órganos consecutivos dividiéndolos en 2 grupos (donación de paciente vivo o donación cadavérica) y se realizaron determinaciones sanguíneas de niveles de interleuquinas, pudo observarse cómo los niveles de TNF- $\alpha$, interleuquina-2R, interleuquina- 6 e interleuquina-8 estaban significativamente más elevados en aquellos donantes en muerte encefálica. Estos datos indican que ya antes de la realización del procedimiento quirúrgico del trasplante 
renal en humanos hay una diferencia entre los órganos provenientes en donantes vivos y los órganos de donantes fallecidos en relación con su contenido en citoquinas.

No solamente diversos autores constataron la elevación de los niveles plasmáticos de citoquinas así como de las alteraciones histoquímicas que se producen en el riñón tras la muerte encefálica, sino que también se estudió su relación con la disfunción del injerto renal. Nijboer et $\mathrm{al}^{26}$ realizaron biopsias renales mediante aguja a riñones obtenidos de muerte encefálica y de donantes vivos. En las biopsias practicadas a riñones de donantes en muerte encefálica se evidenció un significativo incremento en la presencia de leucocitos en el intersticio, aunque no en el glomérulo, e igualmente la muerte encefálica se acompañó de un significativo incremento de E-selectina; por el contrario, la expresión de ICAM-1 y VCAM-1 fue similar en ambos grupos. Cuando se evaluó la expresión génica de proteínas de choque térmico (heat shock proteins), se observó que tras la muerte encefálica la expresión génica de HO-1 y Hsp-70 se triplicaba en el grupo de donantes en muerte encefálica en comparación con el de donantes vivos. El estudio global de la posible relación de la respuesta inflamatoria y de las proteínas de estrés, expresadas en el riñón proveniente de donante en muerte encefálica, mostró en este grupo de pacientes que la alta expresión intersticial de ICAM-1 y de VCAM-1 se mostró como un factor de riesgo para el desarrollo de función retardada del injerto así como para la elevación de la creatinina en la evolución al primer y al tercer año después del trasplante.

\section{Influencia de la muerte encefálica sobre otros órganos intraabdominales}

La influencia de la muerte encefálica también se ha analizado en otros órganos intraabdominales, como el hígado $^{27}$ y el intestino, y se han detectado importantes cambios histológicos e inflamatorios con potenciales consecuencias tras el trasplante.

Van der Hoeven et $\mathrm{al}^{28}$, mediante estudios en animales de modelos de muerte encefálica, estudiaron el efecto de ésta sobre el hígado (grupo de animales en muerte encefálica con hipotensión, grupo de muerte encefálica con normotensión y grupos control) y pudieron confirmar no sólo la existencia de hallazgos patológicos en el hígado de los animales en muerte encefálica, sino también la influencia que la hipotensión podía tener sobre esta respuesta. Los mencionados autores analizaron, mediante tinción inmunohistoquímica, la expresión endotelial de ICAM-1 y de VCAM-1, que no se evidenciaron en el estudio histoquímico del grupo control; por el contrario, la VCAM-1 estuvo presente en las células endoteliales de las venas centrales de los grupos de muerte encefálica. La expresión de ICAM-1 estuvo significativamente incrementada en los grupos de muerte encefálica (tanto con hipotensión como con normotensión) en comparación con el grupo control. La infiltración leucocitaria en el tejido hepático también fue un fenómeno asociado a la muerte encefálica, tanto en el donante normotenso como en el donante con hipotensión: ya desde la primera hora tras la muerte encefálica se observó un incremento de los infiltrados leucocitarios. El elemento diferencial entre el grupo con hipotensión y el grupo con normotensión fue que en éste los infiltrados de linfocitos $\mathrm{T}$ fueron significativamente más prominentes en el primer grupo. Este mismo estudio también evidenció que la muerte encefálica se acompaña de un progresivo incremento de infiltrados de polimorfonucleares en el parénquima hepático, un efecto que fue progresivo a partir de la instauración de la muerte encefálica. En consecuencia, puede inferirse que la fase agonal en presencia de muerte encefálica induce una cascada de episodios que determinan una significativa activación de la respuesta celular inmunitaria, que puede potenciarse por la presencia de factores tales como la hipotensión arterial. La progresiva disfunción hepática, junto con la infiltración linfocitaria con aumento de la inmunogenicidad del potencial donante de órganos, puede afectar la viabilidad del injerto ${ }^{29}$.

Investigaciones como las de Koudstaal et $\mathrm{al}^{30}$ también han demostrado las consecuencias de la muerte encefálica sobre el intestino del donante y la respuesta inflamatoria subsecuente a ella. Estos autores estudiaron cambios inflamatorios y apoptóticos en el intestino de animales a los que se les había producido una muerte encefálica en comparación con un grupo control de animales. Un incremento de la concentración de polimorfonucleares en la primera y la cuarta hora después de la muerte encefálica pudo observarse en el grupo en muerte encefálica en comparación con el grupo control. Las ICAM-1, las VCAM-1, las E-selectinas y la interleuquina-6 estuvieron sobrerreguladas también en ese mismo período. Células caspasa-3 positivas se encontraron en el yeyuno y el íleon del grupo de muerte encefálica en la cúpula de las microvellosidades. A nivel plasmático también se observó un significativo incremento de los niveles de interleuquina- 6 en el grupo de muerte encefálica. Estos datos demuestran la inmediata influencia que el desarrollo de muerte encefálica tiene sobre los procesos de apoptosis e inflamación en el intestino, lo que, al igual que ocurre con otros órganos, podría suponer un factor de riesgo en la evolución tras el trasplante intestinal.

\section{Protección de los órganos antes de la extracción. Una nueva estrategia terapéutica}

Las múltiples evidencias que sustentan que la respuesta inflamatoria en el donante de órganos puede tener una influencia negativa en la evolución del injerto apoyan el hecho de que deban realizarse estrategias de protección de estos órganos no solamente después del trasplante, sino también en la fase en el período in vivo antes de la extracción de órganos. Además del mantenimiento de la estabilidad hemodinámica en rangos de presión arterial media adecuados para la edad y los antecedentes del donante, se han propuesto estrategias farmacológicas que podrían aminorar la respuesta inflamatoria inducida por la muerte encefálica. En un estudio ${ }^{31}$ dirigido a conocer la expresión de citoquinas proinflamatorias (se comparó suero y expresión tisular) en donantes fallecidos en muerte encefálica en comparación con donantes vivos así como el efecto que el tratamiento de los donantes con esteroides podía tener en esta respuesta inflamatoria, pudo observarse que inmediatamente después de la laparotomía, la transcripción de proteínas inflamatorias así como de 
citoquinas antiinflamatorias (interleuquina-6, interleuquina10, $\mathrm{CD} 3$, factor de crecimiento de transformación beta (TGFB), factor de necrosis tumoral alfa (TNF- $\alpha$ ), associate athanogene (BAG) y heme oxygenase (HO-1) estaba significativamente elevada en los órganos de muertos encefálicos en comparación con los órganos de donantes vivos. Además de ello, un grupo adicional de donantes fallecidos se trató con esteroides antes de realizar la extracción de órganos. El tratamiento con esteroides disminuyó significativamente la expresión sérica de citoquinas proinflamatorias en comparación con los niveles observados en los donantes vivos.

Recientes estudios han tratado de investigar si el tratamiento con metilprednisolona aplicado al donante en muerte encefálica se acompañaba de cambios en la evolución del ulterior trasplante hepático ${ }^{32}$. Para eso, se realizó un estudio aleatorizado, en el que se aplicaron tratamientos con corticoides a un grupo de donantes en muerte encefálica y se compararon los resultados con los del grupo control de donantes, en el que el tratamiento esteroideo no se aplicó. Se realizaron biopsias hepáticas y se tomaron muestras séricas inmediatamente antes de la laparotomía. La evaluación de los niveles de citoquinas en suero antes de la extracción hepática reveló que los marcadores inflamatorios (interleuquina-6, TNF- $\alpha$, interleuquina-2 e interleuquina-2R- $\alpha$ ) presentaban unas concentraciones significativamente inferiores en el grupo tratado en comparación con el grupo de donantes no tratado. Igualmente, con el objeto de conocer si la disminución de la activación de la respuesta inmunitaria del injerto debida al tratamiento con esteroides podía tener un impacto en la evolución final del injerto, se realizó una comparación de los niveles de alanina aminotransferasa (ALT) y aspartato aminotransferasa (AST) (como marcadores de la lesión por isquemia/reperfusión), gamma GT (como marcador de lesión biliar) y bilirrubina (como marcador de función hepática). Los resultados mostraron que los niveles plasmáticos de estos marcadores eran significativamente más bajos en el grupo con tratamiento esteroideo que en el grupo control. Los órganos provenientes de donantes no tratados presentaron una mayor tasa de rechazo agudo (evidenciado mediante biopsia hepática) a los 6 meses después del trasplante que el grupo tratado. Este estudio aporta una alta evidencia de que el tratamiento con metilprednisolona en el donante hepático tiene una influencia positiva en los resultados del trasplante, posiblemente mediada por la disminución de la respuesta inflamatoria que se produce debida a la muerte encefálica.

Además del tratamiento con esteroides, se están proponiendo otros tipos de fármacos con capacidad inmunomoduladora como opciones terapéuticas en el donante a fin de mejorar los resultados del trasplante. Hoeger et $\mathrm{al}^{33}$ instauraron, en un modelo experimental en ratas, tratamiento con dopamina a ratas donantes renales en muerte encefálica y a un grupo control que no recibió este tratamiento. Tras el trasplante de esos riñones, se pudo observar que los niveles de creatinina y el grado de infiltración leucocitaria eran significativamente menores en el grupo que recibió tratamiento con dopamina. En este estudio, la expresión del mARN de la interleuquina estuvo significativamente disminuida en el grupo tratado con dopamina. Estos hallazgos pueden explicar el potencial efecto beneficioso de la utilización de dopamina en el tratamiento del donante renal. Parece, por tanto, que el efecto beneficioso del tratamiento con dopamina en el donante, además de por su efecto de estabilización hemodinámica, puede también estar mediado por un efecto directo antiinflamatorio mediante activación de los receptores-D dopaminérgicos.

\section{Conclusiones}

El estado de muerte encefálica debe considerarse un estado patológico no solamente para el cerebro, sino también para el resto del organismo. La muerte encefálica genera respuestas hemodinámicas, endocrinas e inflamatorias que afectan gravemente a los órganos del donante cadáver. Esa afectación de los órganos tras la muerte encefálica puede tener consecuencias relevantes en el resultado de los trasplantes con estos órganos. En consecuencia, el conocimiento de la fisiopatología de la respuesta orgánica y sistémica a la muerte encefálica, principalmente de la respuesta inflamatoria, puede ser de gran trascendencia para incrementar el éxito de los trasplantes de órganos sólidos. El desarrollo del área de conocimiento de la neuroinmunomodulación así como la realización de tratamientos específicos para control de la cascada inflamatoria tras la muerte encefálica abren la posibilidad de mejorar la supervivencia de los órganos y de los pacientes que reciben un trasplante.

\section{Bibliografía}

1. Terasaki PI, Cecka JM, Gjertson DW, Takemoto S. High survival rates of kidney transplants from spousal and living unrelated donors. N Engl J Med. 1995;10:333-6.

2. Chertow GM, Milford EL, Mackenzie HS, Brenner BM. Antigenindependent determinants of cadaveric kidney transplant failure. JAMA. 1996;276:1732-6.

3. Orloff MS, Reed Al, Erturk E, Kruk RA, Paprocki SA, Cimbalo SC, et al. Nonheartbeating cadaveric organ donation. Ann Surg. 1994;220:578-83.

4. Murugan R, Venkataraman R, Wahed AS, Elder M, Hergenroeder $G$, Carter $M$, et al. Increased plasma interleukin- 6 in donors is associated with lower recipient hospital-free survival after cadaveric organ transplantation. Crit Care Med. 2008;36:1810-16.

5. Barklin A, Larsson A, Vestergaard C, Koefoed-Nielsen J, Bach A, Nyboe R, et al. Does brain death induce a pro-inflammatory response at the organ level in a porcine model?. Acta Anaesthesiol Scand. 2008;52:621-7.

6. Amado JA, López-Espadas F, Vázquez-Barquero A, Salas E, Riancho JA, López-Cordovilla JJ, et al. Blood levels of cytokines in brain-dead patients: Relationship with circulating hormones and acute-phase reactants. Metabolism. 1995;44:812-16.

7. Kusaka M, Pratschke J, Wilhelm MJ, Ziai F, Zandi-Nejad K, Mackenzie HS, et al. Activation of inflammatory mediators in rat renal isografts by donor brain death. Transplantation. 2000;69:405-10.

8. Goes N, Urmson J, Ramassar V, Halloran PF. Ischemic acute tubular necrosis induces an extensive local cytokine response. Evidence for induction of interferon-gamma, transforming growth factor-beta 1, granulocyte-macrophage colony-stimulating factor, interleukin-2, and interleukin-10. Transplantation. 1995;59:565-72.

9. Novitzky D, Cooper DK, Muchmore JS, Zuhdi N. Pituitary function in brain-dead patients. Transplantation. 1989;48: 1078-1079. 
10. Ojeda R, Cerro S, Ordonez F, Dominguez-Roldan JM, Hernandez F. El propranolol protege el miocardio y previene la hipotensión arterial en un modelo experimental de donante de órganos. Rev Esp Anestesiol Reanim. 2002;49:229-37.

11. Novitzky D, Rhodin J, Cooper DK, Ye Y, Min KW, DeBault L. Ultrastructure changes associated with brain death in the human donor heart. Transpl Int. 1997;10:24-32.

12. Shivalkar B, Van Loon J, Wieland W, Tjandra-Maga TB, Borgers $M$, Plets $C$, et al. Variable effects of explosive or gradual increase of intracranial pressure on myocardial structure and function. Circulation. 1993;87:230-9.

13. Mertes PM, Carteaux JP, Jaboin Y, Pinelli G, el Abassi K, Dopff C, et al. Estimation of myocardial interstitial norepinephrine release after brain death using cardiac microdialysis. Transplantation. 1994;57:371-7.

14. Ferrera R, Ovize M, Claustrat B, Hadour G. Stable myocardial function and endocrine dysfunction during experimental brain death. J Heart Lung Transplant. 2005;24:904-11.

15. Segel LD, VonHaag DW, Zhang J, Follette DM. Selective overexpression of inflammatory molecules in hearts from brain-dead rats. J Heart Lung Transplant. 2002;21:804-11.

16. Zweers N, Petersen AH, Van der Hoeven JA, De Haan A, Ploeg RJ, De Leij LF, et al. Donor brain death aggravates chronic rejection after lung transplantation in rats. Transplantation. 2004;78:1251-8.

17. López-Aguilar J, Villagrá A, Bernabé F, Murias G, Piacentini E, Real $\mathrm{J}$, et al. Massive brain injury enhances lung damage in an isolated lung model of ventilator-induced lung injury. Crit Care Med. 2005;33:1077-83.

18. Avlonitis VS, Wigfield CH, Kirby JA, Dark JH. The hemodynamic mechanisms of lung injury and systemic inflammatory response following brain death in the transplant donor. Am J Transplant. 2005;5:684-93.

19. Edmonds Jr HL, Cannon Jr HC, Garretson HD, Dahlquist G. Effects of aerosolized methylprednisolone on experimental neurogenic pulmonary injury. Neurosurgery. 1986;19: $36-40$.

20. Minnear FL, Connell RS. Prevention of aconitine-induced neurogenic pulmonary edema (NPE) with hypovolemia or methylprednisolone. J Trauma. 1982;22:121-8.

21. Fisher AJ, Donnelly SC, Hirani N, Haslett C, Strieter RM, Dark $\mathrm{JH}$, et al. Elevated levels of interleukin-8 in donor lungs is associated with early graft failure after lung transplantation. Am J Respir Crit Care Med. 2001;163:259-65.

22. Locke JE, Segev DL, Warren DS, Dominici F, Simpkins CE, Montgomery RA. Outcomes of kidneys from donors after cardiac death: Implications for allocation and preservation. Am J Transplant. 2007;7:1797-807.

23. Pelletier Shawn J, Merion Robert M. Implications and outcomes of donors after cardiac death. Current Opinion in Organ Transplantation. 2007;12:125-30.

24. Koo DD, Welsh KI, McLaren AJ, Roake JA, Morris PJ, Fuggle SV. Cadaver versus living donor kidneys: Impact of donor factors on antigen induction before transplantation. Kidney Int. 1999;56:1551-9.

25. Stangl M, Zerkaulen T, Theodorakis J, Illner W, Schneeberger H, Land $\mathrm{W}$, et al. Influence of brain death on cytokine release in organ donors and renal transplants. Transplant Proc. 2001;33: 1284-1285.

26. Nijboer WN, Schuurs TA, Van der Hoeven JA, Leuvenink HG, Van der Heide JJ, Van Goor H, et al. Effects of brain death on stress and inflammatory response in the human donor kidney. Transplant Proc. 2005;37:367-9.

27. Kwekkeboom J, Kuijpers MA, Bruyneel B, Mancham S, De BaarHeesakkers E, ljzermans JN, et al. Expression of CD80 on Kupffer cells is enhanced in cadaveric liver transplants. Clin Exp Immunol. 2003;132:345-51.

28. Van der Hoeven JA, Ter Horst GJ, Molema G, De Vos P, Girbes AR, Postema F, et al. Effects of brain death and hemodynamic status on function and immunologic activation of the potential donor liver in the rat. Ann Surg. 2000;232: 804-13.

29. Zhang S, Zhu C, Wu Y, Zhai W, Shi J, Song Y, et al. Brain death affects the hepatic immunogenicity of pigs. Transplant Proc. 2008;40:2159-62.

30. Koudstaal LG, 't Hart NA, Ottens PJ, Van den Berg A, Ploeg RJ, Van Goor $\mathrm{H}$, et al. Brain death induces inflammation in the donor intestine. Transplantation. 2008;86:148-54.

31. Kuecuek O, Mantouvalou L, Klemz R, Kotsch K, Volk HD, Jonas S, et al. Significant reduction of proinflammatory cytokines by treatment of the brain-dead donor. Transplant Proc. 2005;37:387-8.

32. Kotsch K, Ulrich F, Reutzel-Selke A, Pascher A, Faber W, Warnick $P$, et al. Methylprednisolone therapy in deceased donors reduces inflammation in the donor liver and improves outcome after liver transplantation: A prospective randomized controlled trial. Ann Surg. 2008;248:1042-50.

33. Hoeger S, Reisenbuechler A, Gottmann U, Doyon F, Braun C, Kaya Z, et al. Donor dopamine treatment in brain dead rats is associated with an improvement in renal function early after transplantation and a reduction in renal inflammation. Transpl Int. 2008;21:1072-80. 